\title{
Lymphatic tuberculosis after lymphadenectomy for ovarian cancer: a case report
}

\author{
Shuang Sheng, Bo Chi, Yan Kuang \\ Department of Gynecology, The First Affiliated Hospital of Guangxi Medical University, Nanning, China \\ Correspondence to: Yan Kuang. Department of Gynecology, The First Affiliated Hospital of Guangxi Medical University, Nanning, China. \\ Email: kuangyan_2004@163.com.
}

\begin{abstract}
Backgroundk We present the case of one patient with early ovarian cancer complicated with lymphatic tuberculosis (TB) and discuss the significance of lymph node resection for ovarian cancer. We also reflect on the limitations of the preoperative imaging evaluation of lymph node metastasis and suggest possible solutions. The clinical data of a patient with early ovarian cancer complicated with lymphatic TB were analyzed retrospectively.
\end{abstract}

Case Description: A 37-year-old woman was diagnosed with a left ovarian malignant tumor and multiple pelvic and abdominal lymph node metastases using full abdominal computed tomography (CT) and 18-fluorodeoxyglucose-positron emission tomography/computed tomography $\left({ }^{18} \mathrm{FDG}-\mathrm{PET} / \mathrm{CT}\right)$ before surgery. Intraoperative frozen pathology findings suggested an adult-type granulosa cell tumor (AGCT). Transabdominal debulking surgery and lymph node dissection were performed. Routine pathological results suggested that the tumor was confined to the left ovary and the lesion in the pelvis and abdomen was lymph node TB. The Federation International of Gynecology and Obstetrics (FIGO) pathological stage was IA1, so lymph node dissection was unnecessary. After discharge, the patient received anti-TB drugs. She recovered without bleeding, lymphatic cyst, lymphedema, or other surgery-related complications.

Conclusions: Clinicians should, therefore, avoid relying exclusively on imaging results. Instead, it is necessary to make comprehensive decisions based on a combination of the patients' medical history, intraoperative condition, and histopathological type. If necessary, clinicians should sample suspicious and/or increased lymph nodes and send them for intraoperative frozen pathological examination. Excessive surgical treatment and early misdiagnosis in ovarian cancer should be avoided.

Keywords: Ovarian cancer; adult granulocytoma; lymph node dissection; lymph node tuberculosis; case report

Submitted Dec 10, 2021. Accepted for publication Feb 16, 2022.

doi: $10.21037 /$ gs-22-8

View this article at: https://dx.doi.org/10.21037/gs-22-8

\section{Introduction}

Ovarian cancer is a common gynecological malignant tumor. It ranks first among female reproductive system tumors for mortality and lacks specific clinical manifestations in its early stages. A total of $70-75 \%$ of patients are in the advanced stages at the time of diagnosis (1). Surgery is the main treatment for patients with ovarian cancer.

The three major metastatic pathways of ovarian cancer are direct spread, intraperitoneal implantation, and lymphatic metastasis. Its metastasis is characterized by extensive pelvic and intraperitoneal metastases, including retroperitoneal lymph node metastases. It has been reported that the probabilities of finding lymph node metastases in the early and late stages of ovarian cancer are $13-20 \%$ and $50 \%$, respectively. The prognosis is mainly related to the location and size of lymph node metastasis. Involvement of para-aortic lymph nodes with malignant cells along ovarian vessels is the earliest route of lymphatic spread, followed by pelvic and inguinal lymph nodes. Involvement of paraaortic lymph nodes only in advanced stages has a better prognosis compared with pelvic lymph node involvement 
or involvement of both systems, and tumor size less than or equal to $10 \mathrm{~mm}$ has better survival benefit (2). Lymphadenectomy will increase the incidence of surgeryrelated complications, including lymphatic cysts, lower limb edema, lymphatic leakage, and vascular disease. It will also increase the treatment burden and affect the patients' quality of life. There has, therefore, been extensive controversy regarding lymphadenectomy for ovarian cancer.

It is currently recommended that pelvic and paraaortic lymphadenectomy be performed in patients with earlystage ovarian cancer (IA-IIA), that the status of lymph node metastasis be clarified, and that surgical-pathological staging be completed to provide a basis for the formulation of a postoperative adjuvant treatment plan. There are some debates about the therapeutic value of retroperitoneal lymphadenectomy for epithelial early-stage ovarian cancer (ESOC). In a multi-center retrospective cohort study conducted by Nicolò Bizzarri, it was found that pelvic and para-aortic lymphadenectomy in surgical staging of ESOC improves the 5-year disease-free survival (DFS), but no difference in 5-year overall survival (OS) (3). In another retrospective analysis carried out by Ozlem Ercelep in 378 patients, reported that patients of ESOC in stage I-II had no difference in progression free survival (PFS) and OS (4).

It is also recommended that enlarged and/or suspicious lymph nodes be removed as much as possible after maximizing debulking surgery for advanced ovarian cancer, while clinically negative lymph nodes need not be removed. According to the results of the Lymphadenectomy in Ovarian Neoplasms (LION) trial, in patients with advanced ovarian cancer who had undergone R0 resection, systematic pelvic and para-aortic lymphadenectomy did not prolong OS or PFS if the lymph nodes were negative (5). Lymph node resection cannot be performed for histopathological types such as malignant cord-stromal tumors, borderline tumors that can preserve fertility, and early malignant germ cell tumors $(6,7)$.

In conclusion, residual lesions and lymph node metastasis are important factors affecting the prognosis of advanced ovarian cancer (7). Regardless of whether lymph nodes are removed, the purpose of surgery in patients with advanced ovarian cancer is to minimize tumors as much as possible, including pelvic, abdominal, and retroperitoneal lesions, to obtain better prognoses.

This paper discusses the significance of lymph node resection for ovarian cancer from another perspective, reflects on the limitations of preoperative imaging evaluation of lymph node metastasis, and suggests possible solutions. We present the following article in accordance the CARE reporting checklist (available at https:// gs.amegroups.com/article/view/10.21037/gs-22-8/rc).

\section{Case presentation}

According to hospital records, a 37-year-old female patient, G5P2A3, was hospitalized, on Feb 1st, 2021, for menopause for more than 1 year and pelvic mass found 11 months prior to presentation at the clinic. The patient, who usually had regular menstruations, had last menstruated on January 1, 2021. In April 2020, she had gone to hospital for gynecological ultrasonography due to menopause for more than 3 months, and a cyst in the left adnexal area (approximately $4.4 \mathrm{~cm}$ in size) had been identified, but left untreated. The patient had fertility issues and went to the First Affiliated Hospital of Guangxi Medical University in January 2021 for gynecological B-ultrasound, which showed an inner membrane of $2 \mathrm{~mm}$, and a $68 \mathrm{~mm} \times 53 \mathrm{~mm} \times$ $44 \mathrm{~mm}$ mixed echo mass at the left accessory area with internal hypoechoic and irregular liquid dark areas of different sizes. Given the possibility of an ovarian granulosa cell tumor, she was admitted to the gynecological ward of the First Affiliated Hospital of Guangxi Medical University for further treatment.

The patient had no abdominal distension, abdominal pain, low fever, night sweat, weight loss, fatigue, or other discomfort. She denied a history of tuberculosis (TB) contact. There was no fever at admission, no abnormalities were found in the heart and lung examinations, and the shifting dullness test was negative. A gynecological examination suggested that a $6 \mathrm{~cm} \times 6 \mathrm{~cm} \times 6 \mathrm{~cm}$ mass could be palpated in the left accessory area, with medium texture, poor mobility, unclear boundary, and no obvious tenderness. No mass was palpated in the right accessory area, and there was no abnormality observed using a vaginal speculum. The full blood routine test results, blood electrolytes, and hepatic and renal functions were normal, and a quantitative human immunodeficiency virus (HIV) antibody test was negative. The results were as follows: alpha-fetoprotein (AFP), $20.06 \mathrm{ng} / \mathrm{mL}$ (reference range, $0.89-8.78 \mathrm{ng} / \mathrm{mL}$ ); and CA125, CA199, CA153, carcinoembryonic antigen (CEA), human epididymis secretory protein 4 (HE4), $\beta$-humanchorionic gonadotropin ( $\beta$-HCG), protein induced by vitamin $\mathrm{K}$ absence-II (PIVKA-II), and other tumor markers were within the laboratory's normal ranges. The levels of six sex hormones were as follows: Follicle-stimulating hormone (FSH), $4.20 \mathrm{mIU} / \mathrm{mL}$; luteinizing hormone (LH), 

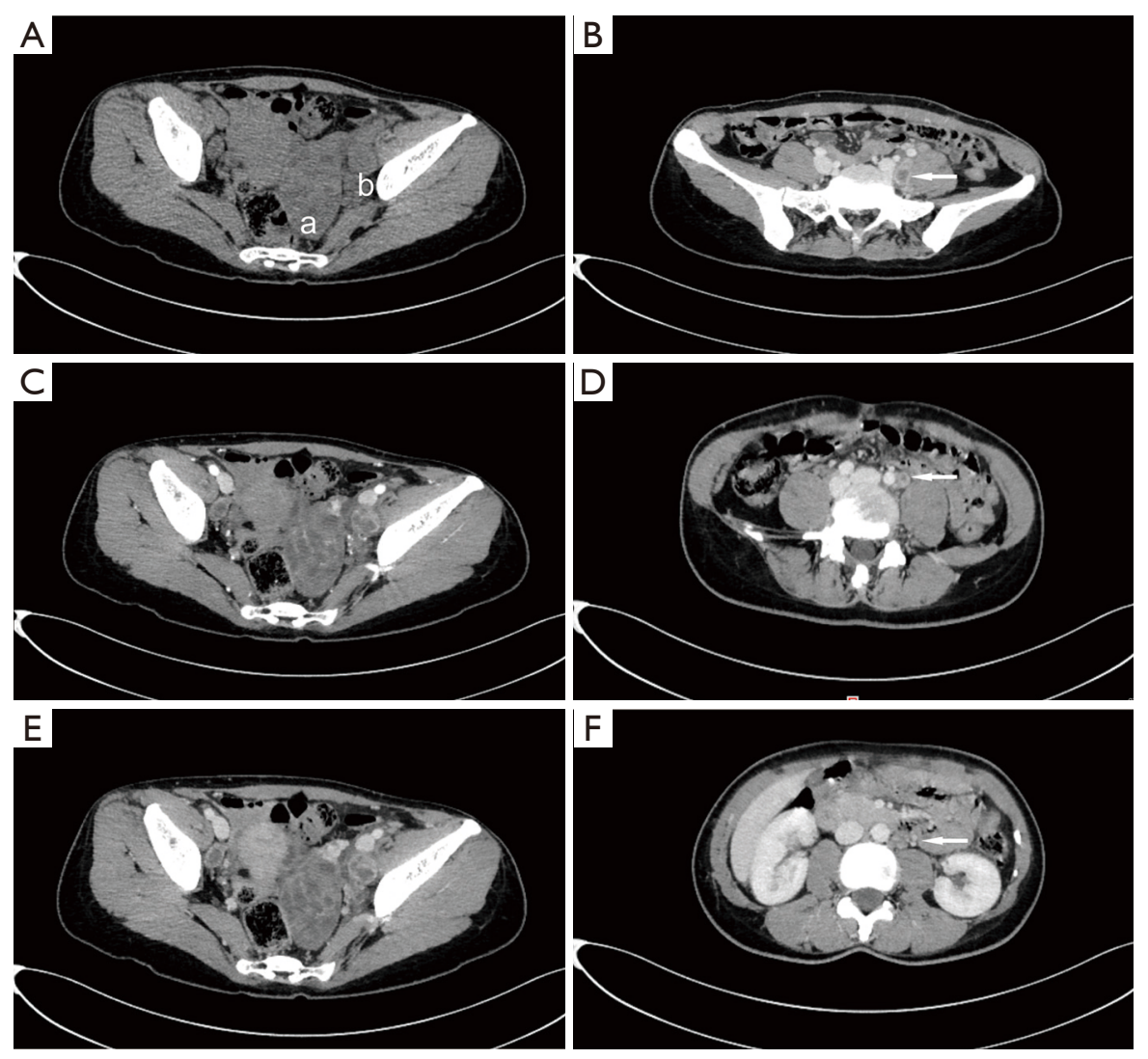

Figure 1 CT scan of the patient's pelvis and abdomen. (A) Plain CT scan of a mass in the left adnexal area (a: mass in the left adnexal area; b: left pelvic lymph node); (B) CT arterial phase of a mass in the left adnexal area; (C) CT venous phase of a mass in the left adnexal area; (D) pelvic lymph node; (E) left para-iliac lymph node; (F) para-aortic lymph node. CT, computed tomography.

$37.61 \mathrm{mIU} / \mathrm{mL}$; prolactin (PRL), $23.13 \mathrm{ng} / \mathrm{mL}$; progesterone $(\mathrm{P}), 0.65 \mathrm{ng} / \mathrm{mL}$; testosterone $(\mathrm{T})$, $1.49 \mathrm{ng} / \mathrm{mL}$, and estradiol (E2), $32.23 \mathrm{pg} / \mathrm{mL}$.

Plain and enhanced computed tomography (CT) scans of the chest, whole abdomen, and pelvic cavity indicated that the left adnexal area showed a mass of $7.6 \mathrm{~cm} \times 4.6 \mathrm{~cm}$ $\times 4.6 \mathrm{~cm}$ (Figure 1). We considered it to be a left ovarian malignant tumor, or possibly a granulosa cell tumor or cystadenocarcinoma. No abnormality was found in the right ovary or uterus. There was lymph node metastasis in the paraaortic, pelvic, and bilateral inguinal areas. The largest metastasis was approximately $2.8 \mathrm{~cm} \times 1.9 \mathrm{~cm}$, and liquefied necrotic areas were seen in some parts (Figure 1). Plain and enhanced CT scans of the liver, gallbladder, pancreas, and spleen showed no abnormality and no peritoneal effusion. There was no exudation, cavity, hilar lymph node enlargement, or pleural effusion in the lungs. To exclude a gastrointestinal malignant tumor, painless gastroenteroscopy was performed, and there were no special findings. Because the CT indicated multiple lymph node metastases in the pelvic and abdominal cavities, whole-body positron emission tomography (PET)-CT was conducted to further characterize the scope of tumor metastasis. The results showed that there was an irregular cystic mass on the left side of the pelvic cavity, with a size of $38 \mathrm{~mm}$ $\times 46 \mathrm{~mm} \times 77 \mathrm{~mm}$ (Figure 2), uneven density, uneven glucose metabolism, and a slightly increased maximum standard uptake value (SUVmax) of 1.8. We considered the possibility of a malignant tumor on the left side of the pelvic cavity (possibly a left ovarian granulosa cell tumor or cystadenoma). Multiple enlarged lymph node shadows with increased glucose metabolism were seen near the abdominal aorta (below the L3 plane), the left iliac artery, and the left and right sides of the pelvic cavity. The largest one was approximately $20 \mathrm{~mm} \times 22 \mathrm{~mm}$, with a SUVmax of 7.3. Multiple lymph node metastases were therefore considered (Figures 3-5).

Because the preoperative CT and PET-CT showed 


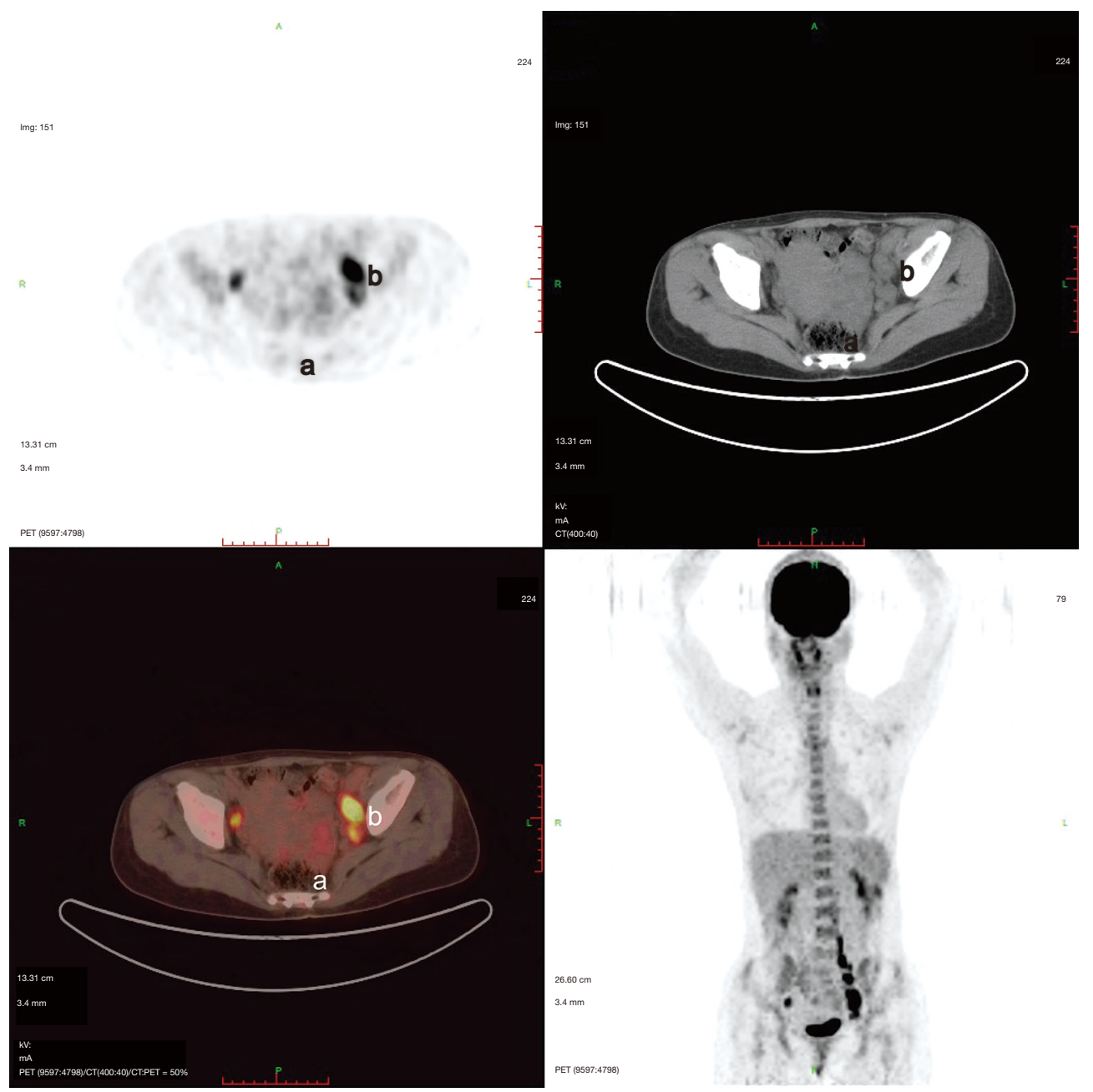

Figure 2 PET/CT scan of a mass in the left adnexal area (a: mass in the left adnexal area; b: left pelvic lymph node). PET/CT, positron emission tomography/computed tomography.

multiple metastases of abdominal paraaortic and pelvic lymph nodes, we considered the possibility of a clinically advanced ovarian cancer. Consequently, an abdominal median longitudinal incision was performed, and laparotomy was conducted. There was no ascites, and no thickening or adhesion of the greater omentum was noted during surgery. The size of the left ovary was approximately $10 \mathrm{~cm} \times 8 \mathrm{~cm} \times 6 \mathrm{~cm}$, the surface was smooth with no breach, and the morphology and appearance of the right ovary and fallopian tube were normal. No tumor seeding lesions were found on the surface of the peritoneal cavity or peritoneum. The abdominal paraaortic lymph nodes, presacral lymph nodes, and bilateral pelvic lymph nodes were palpable, enlarged to different sizes, and some showed beaded changes. The larger ones were approximately $3 \mathrm{~cm}$ $\times 2 \mathrm{~cm} \times 2 \mathrm{~cm}$. The peritoneal lavage fluid was reserved for pathological examination. The left adnexa was completely removed and sent for intraoperative frozen pathological examination together with part of the right ovarian tissue. The results showed a left ovarian adult-type granulosa cell tumor (AGCT). Due to the patient's strong desire to preserve her reproductive functions, the surgeon consulted with the patient's husband about the intraoperative situation and the results of the frozen pathological examinations, to inform him of the patient's condition and possible prognosis. Ovarian tumor cell reduction surgery, i.e., left adnexectomy + right ovarian biopsy + para-aortic lymph node dissection near the main abdominal artery + pelvic lymph node dissection + greater omentum resection, was finally performed to preserve the reproductive functions. 


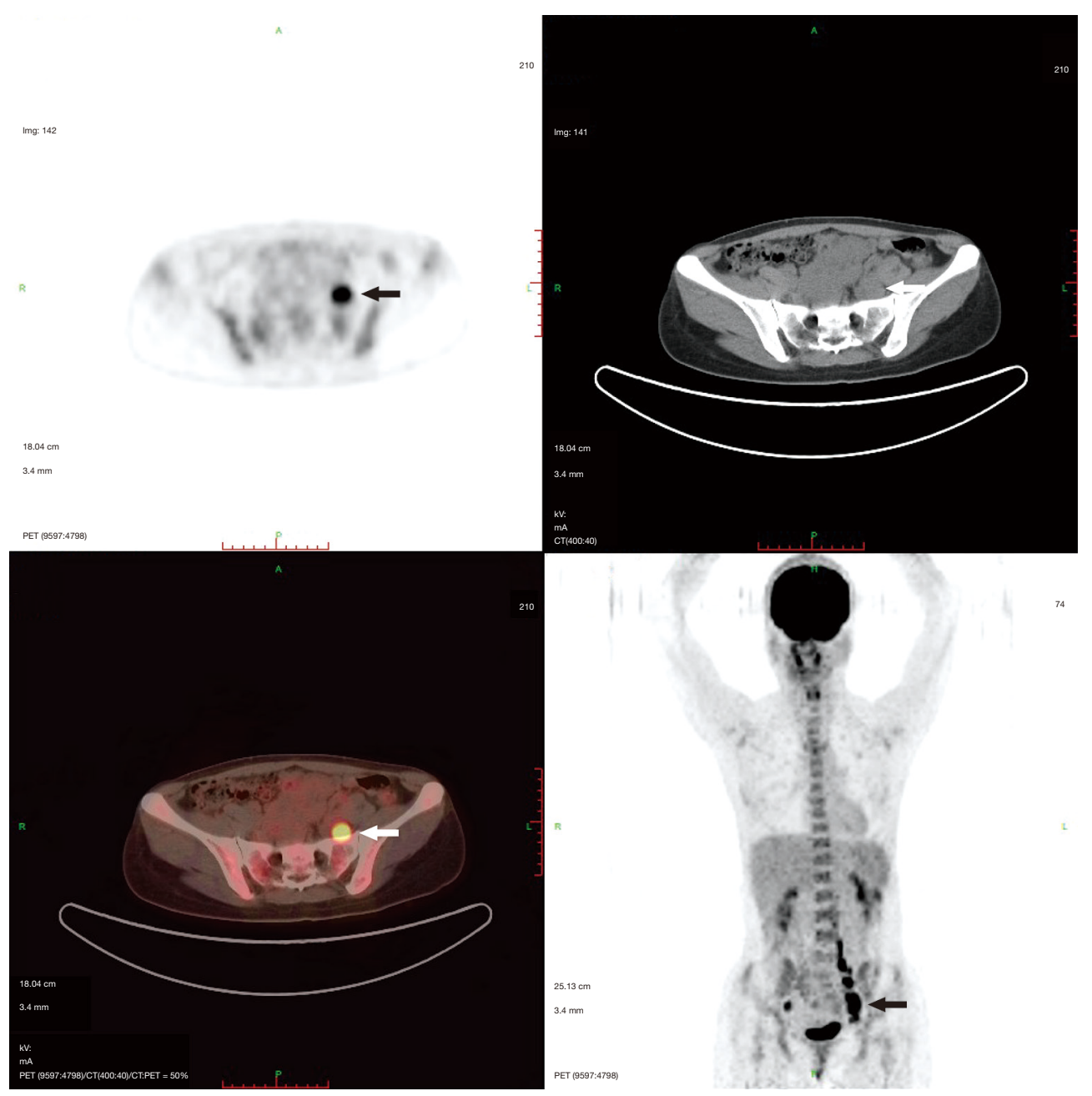

Figure 3 PET/CT scan of the pelvic lymph node. The arrows indicate hypermetabolic foci of lymph nodes. PET/CT, positron emission tomography/computed tomography.

The scope of the lymph node dissection reached $2 \mathrm{~cm}$ above the inferior mesenteric artery, below the deep iliac circumflex vein, outward to the surface of the psoas major muscle, inward to the outer edge of closed umbilical artery and internal iliac vein, and downward to the obturator nerve.

The results of postoperative pathological and immunohistochemical examinations (Figure 6) showed the presence of a left ovarian AGCT, but there was no tumor metastasis in the lymph node tissue, left fallopian tube, right ovarian biopsy tissue, abdominal flushing fluid, or omentum. A total of 30 lymph nodes were cleaned, of which seven showed chronic granulomatous inflammation, cheese-like necrosis, epithelioid cells, and Langerhans giant cells, suggesting the possibility of TB. According to the intraoperative conditions and postoperative pathological examinations, the final diagnosis was (I) a left ovarian granulosa cell tumor (IA1), and (II) lymphatic TB. The patient recovered well after surgery, and luckily there were no surgery-related complications such as bleeding, lymphatic cyst, or lymphedema. After being discharged, the patient went to a specialized hospital and received antituberculous drug treatment. There was no sign of tumor recurrence at a regular follow-up.

All procedures performed in this study were in accordance with the ethical standards of the institutional and/or national research committee(s) and with the Helsinki Declaration (as revised in 2013). Written informed consent was obtained from the patient for publication of this case report and accompanying images. A copy of the written consent is available for review by the editorial office of this journal. 


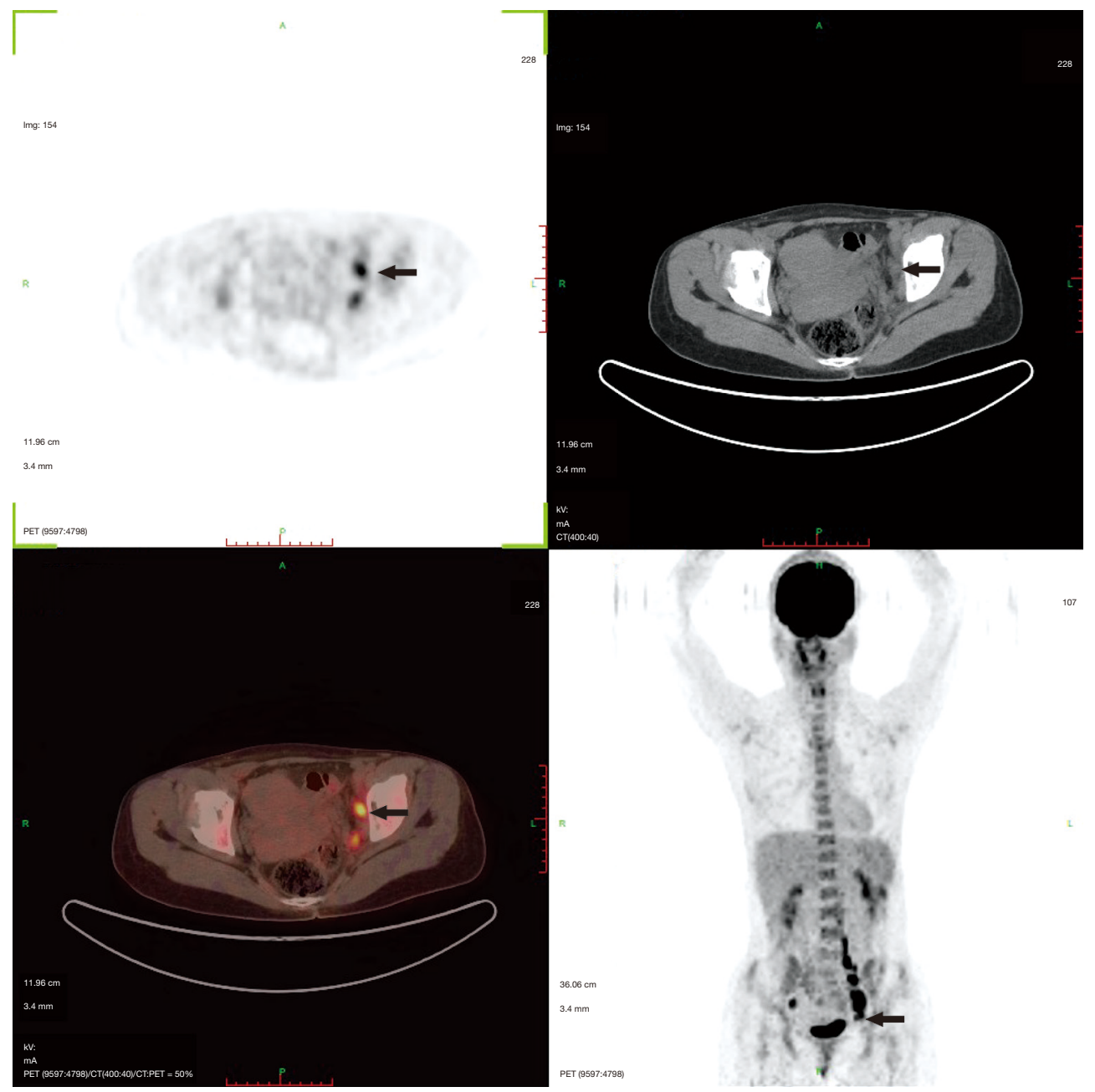

Figure 4 PET/CT scan of the pelvic lymph node. The arrows indicate hypermetabolic foci of lymph nodes. PET/CT, positron emission tomography/computed tomography.

\section{Discussion}

\section{Lymphatic TB}

$\mathrm{TB}$ is one of the major challenges affecting global health. According to the 2020 World Health Organization (WHO) global TB report, approximately 10 million people were infected with $\mathrm{TB}$, and approximately 1.4 million people died of TB worldwide in 2019 (8). TB is divided into two categories, pulmonary TB and extrapulmonary TB (EPTB). EPTB refers to a group of diseases that cause clinical symptoms associated with Mycobacterium tuberculosis, which invades the extrapulmonary organs. In 2019, EPTB accounted for $16 \%$ of new TB cases. The lymph node is the most common site of EPTB, accounting for $35 \%$ of EPTB cases. It often manifests as supraclavicular lymph node enlargement. Mycobacterium tuberculosis reaches the lymph nodes along the lymphatic vessels from the original lesion site (most commonly the lungs), which denotes systemic TB (9).

Lymphoid TB is common in young Asian and African women, especially those with HIV infections. In a large multi-center observational study conducted by Wanli Kang, which collected the epidemiology data of EPTB in China from January 2011 to December 2017, of the 202,998 cases of EPTB, 14,706 (7.24\%) represented tuberculous lymphadenitis of the neck, $1,816(0.89 \%)$ represented pelvic tuberculosis, $1,260(0.62 \%)$ represented tuberculosis of axillary lymph nodes. Symptoms and signs of lymphoid TB vary according to the location of involvement. Patients often lack typical symptoms of TB, such as low fever, night 


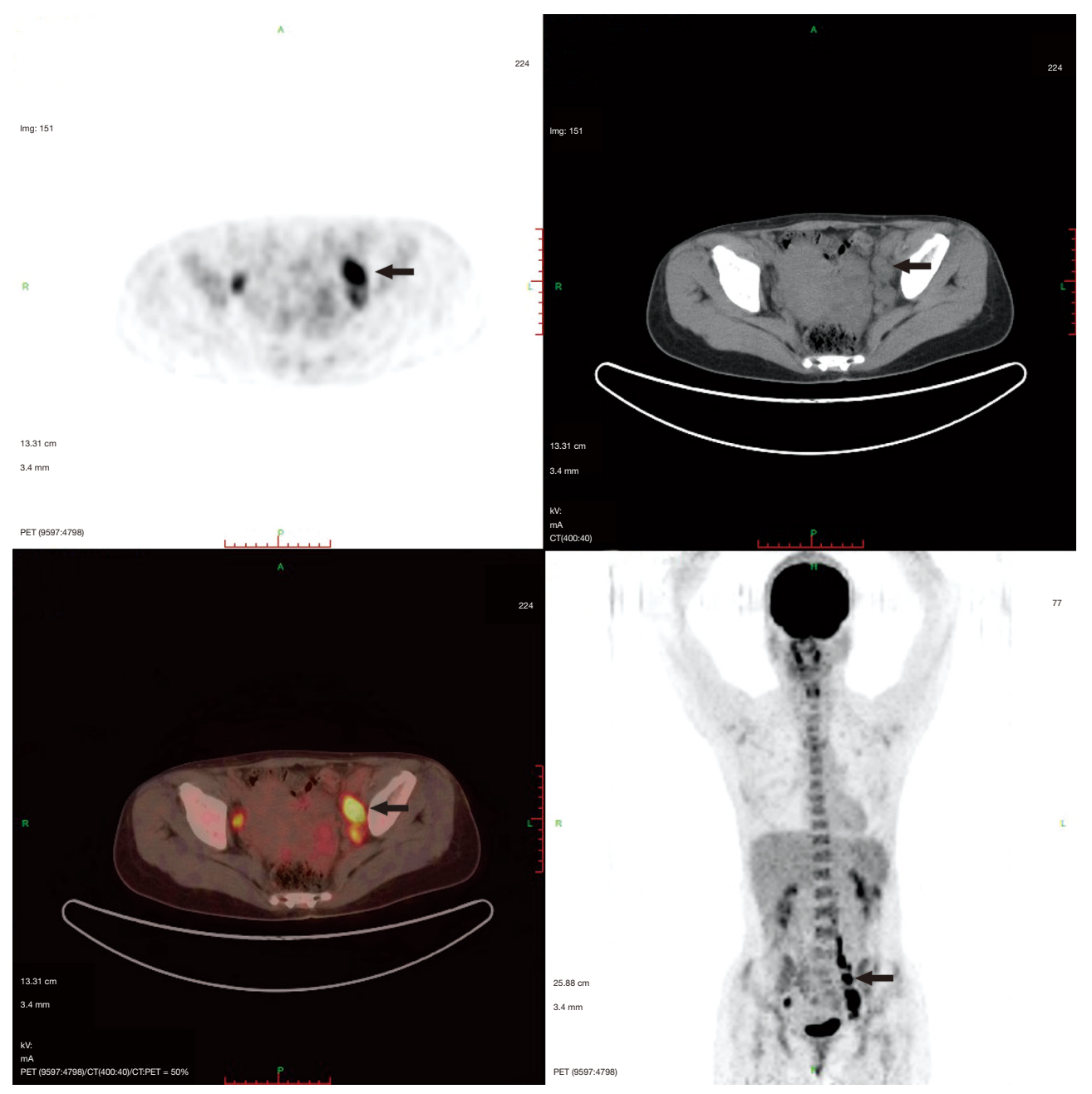

Figure 5 PET/CT scan of the pelvic lymph node. The arrows indicate hypermetabolic foci of lymph nodes. PET/CT, positron emission tomography/computed tomography.

sweats, weight loss, and fatigue. Because most patients deny a history of TB, and there are currently no rapid and effective clinical diagnostic means, early diagnosis is difficult. A definitive diagnosis of tuberculous lymphadenitis can be made by culture or polymerase chain reaction demonstration of $M$. tuberculosis in an affected lymph node. Culture remains the gold standard for diagnosis, but may take 2-4 weeks to yield results (10). Due to the difficulty in obtaining samples and the low bacterial content, the diagnostic sensitivity of lymphoid TB is reduced. However, lymphatic TB should be differentiated from infection, metastasis, nonspecific reactive hyperplasia, sarcoidosis, and connective tissue disease.

Once lymphatic TB is diagnosed, antibiotic treatment should be started in time (9). Surgery is used only in rare cases, such as to obtain a sample for confirmatory testing, for lymph node discomfort that cannot be relieved by medical treatment, or to treat complications associated with lymphatic $\mathrm{TB}$, such as fistula formation and rupture (10).

\section{Imaging evaluation of lymph nodes}

Anti-TB drugs are the first choice for the treatment of lymphatic TB, and lymph node dissection is the first choice to treat lymph node metastasis of ovarian cancer. However, the treatment principles are completely different, so differentiation between the two diseases is particularly important in clinical practice. Lymph node metastasis is an important prognostic factor for ovarian cancer. Determining the location of lymph node involvement is very important 

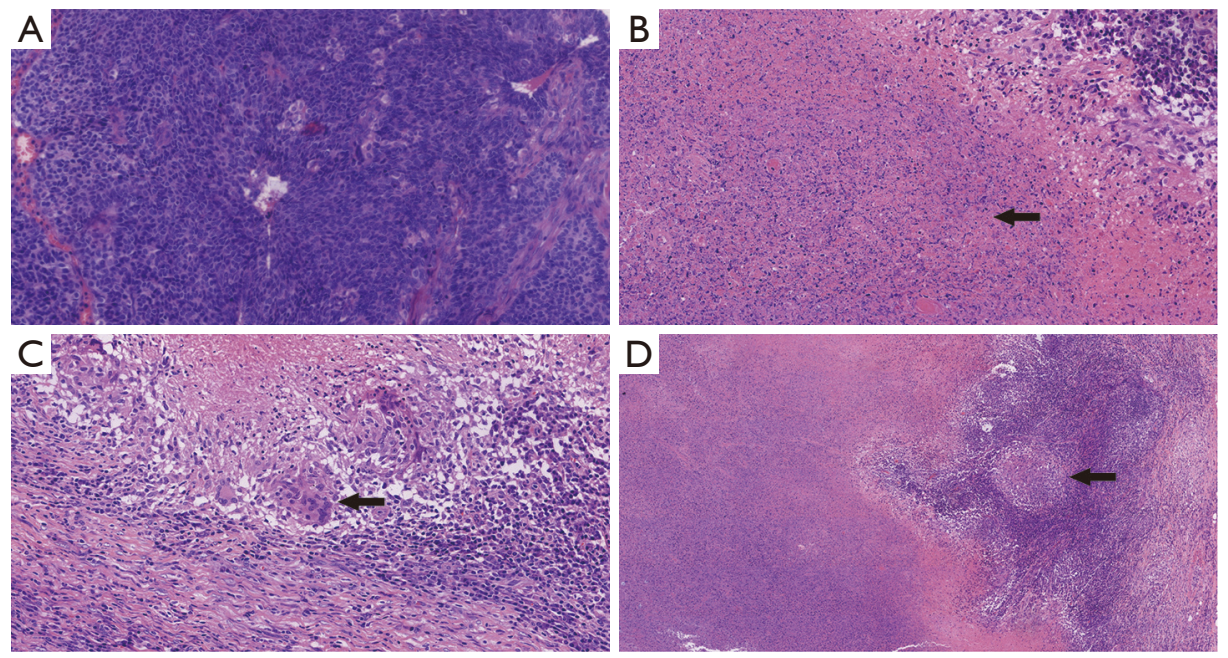

Figure 6 Postoperative routine pathology of the case. (A) HE staining for granulosa cell tumor $(\times 400)$; (B) the arrow indicates caseous necrosis of the lymph nodes $(\times 400)$; $(\mathrm{C})$ the arrows indicate Langhans giant cell (HE staining $\times 400)$; (D) the arrow indicates tuberculous granuloma (HE staining $\times 400)$. HE, hematoxylin-eosin.

for the choice of treatment and the prediction of optimal tumor reduction. Lymph node biopsy is the gold standard for diagnosis, but it increases the incidence of surgical complications. Reliable preoperative imaging evaluation should therefore be conducted to avoid unnecessary lymph node dissection.

As an initial screening method for ovarian cancer, ultrasound is well used in the evaluation of superficial lymph node and FNAC, but its value in the evaluation of deep lymph node status is limited. Morphological imaging methods, such as CT and magnetic resonance imaging (MRI), rely primarily on the size and density of lymph nodes to identify lymph node metastasis of ovarian cancer. These methods usually use a short axis diameter $>8$ or $10 \mathrm{~mm}$ as the main diagnostic criteria (11). However, it is inaccurate to determine lymph node diseases mainly according to size, because lymph nodes of normal size cannot exclude microinfiltration, while enlarged lymph nodes may also show reactive hyperplasia.

To solve the limitations of traditional imaging, molecular imaging methods, such as 18-fluorodeoxyglucose-PET/ CT, have been increasingly used in clinical practice. The imaging principle is based on the uptake ratio of labeled FDG in relation to the glucose metabolizing capacity of tissues, because many malignant tumors have high glucose metabolism levels. It is therefore effective in ovarian cancer staging, lymph node staging, curative effect evaluation, and recurrence evaluation. Compared with traditional imaging, PET/CT can detect smaller lymph node metastases, distant metastases, and co-existing malignant tumors $(11,12)$. The results of a meta-analysis by Yuan $e t$ al. showed that the accuracy of PET-CT in identifying lymph node metastasis of ovarian cancer was better than CT and MRI (12). The sensitivity and specificity of PET$\mathrm{CT}$ and PET in diagnosing metastatic lymph nodes were $73.2 \%$ and $96.7 \%$, respectively, while the sensitivity and specificity of CT were $42.6 \%$ and $95.0 \%$, respectively, and the sensitivity and specificity of MRI were $54.7 \%$ and $88.3 \%$, respectively. Tardieu et al. retrospectively analyzed preoperative PET-CT findings in 63 patients with ovarian cancer (13). The results showed that regardless of the location of the lymph nodes and the patient's treatment plan (surgery after neoadjuvant chemotherapy or initial surgery), PET-CT had high specificity in the preoperative evaluation of lymph node metastasis of advanced epithelial ovarian cancer, for which the specificity for pelvic and inguinal lymph nodes was $91.1 \%$ and $97.6 \%$, respectively. According to the lymphadenectomy in ovarian neoplasms (LION) trial, specificity is an important parameter for lymph node evaluation and can exempt some patients from lymph node resection and reduce the probability of surgical complications.

However, FDG is not a specific tracer for malignant tumors. It can concentrate in some benign lesions and inflammatory cells, such as during inflammation, infection, hysteromyoma, and endometriosis (14). Pathological types 
of TB include proliferative TB, gaseous necrosis, and mixed type TB. Obsolete (older) TB and stable TB usually do not ingest or seldom ingest FDG. PET/CT positive TB foci are hyperplastic and mixed types, because these lesions contain large numbers of epithelial cells, Langerhans giant cells, lymphocytes, and other chronic inflammatory cells (15). The SUV is the most commonly used analysis index in PET examinations. The SUVmax can be used to identify benign and malignant ovarian lesions. SUVmax values differ in different histological types and stages of ovarian cancer (12). Sathekge et al. showed that the SUVmean of lymphatic TB was 6.5 (range, 3.4-9.2), and the SUVmean of malignant lymph nodes was 8.0 (range, 2.5-20.1) (16), and the two overlapped in values. In addition, initial imaging and delayed imaging of lymphatic TB were similar to those of malignant tumors. Therefore, the SUV cutoff value, dual-phase imaging, or delayed imaging cannot accurately distinguish TB from malignant tumors. At present, many second-generation radioactive tracers have been developed and used in clinical practice. It is expected that the increasing use of these ovarian cancer-specific tracers can solve the problems caused by the limitations of FDG.

\section{Limitations}

In the past 30 years, there has been controversy about lymphadenectomy in patients with advanced ovarian cancer. Many previous retrospective studies suggested that patients with advanced ovarian cancer benefited from pelvic and paraaortic lymphadenectomy. Therefore, it has been used as the standard surgery for advanced ovarian cancer for decades. However, there were biases in these retrospective studies, including the scope of lymph node dissection, the size of residual lesions, whether there were suspicious lymph nodes before and during surgery, and the patients' physical conditions. The LION trial results, published in the New England Fournal of Medicine in 2019, prompted gynecological oncologists to re-examine the indications for lymphadenectomy in tumor cell reduction of advanced ovarian cancers (5). The study showed that in patients with advanced ovarian cancer who had undergone R0 resection, systematic pelvic and para-aortic lymph node dissection did not prolong OS or progress-free survival (PFS) if the lymph nodes were negative. On the contrary, this surgery imposed a treatment burden on the patients, and the incidence of postoperative complications was higher. This conclusion was incorporated into the National Comprehensive Cancer Network (NCCN) guidelines in 2019. The guidelines recommended the removal of enlarged or suspicious lymph nodes as much as possible after maximizing tumor reduction for advanced ovarian cancer. Furthermore, lymph node resection was not considered necessary for clinically negative lymph nodes.

Ovarian sex cord-stromal tumors originate from the sex cord and stromal tissue in primitive gonads. Granulosa cell tumor is the most common pathological type, accounting for $5-8 \%$ of ovarian tumors (17). Granulosa cell tumor can be divided into adult- and juvenile-types. The former accounts for $70 \%$ of sex cord-stromal tumors (18). It is a low-grade malignant tumor with good prognosis. The main metastatic modes of sex cord-stromal tumors are direct diffusion and hematogenous metastasis. The probability of lymphatic metastasis is very low. Most recurrent cases show multifocal metastasis. Brown et al. retrospectively analyzed 257 patients with ovarian sex cord-stromal tumors and found that 111 patients had undergone staged surgery (stages I-III) at the initial treatment. Of these, 66 patients had lymph nodes removed, with no tumor metastasis confirmed by pathology (19). The current view is that malignant sex cord-stromal tumors do not require the removal of lymph nodes, unless there are enlarged or suspicious lymph nodes $(6,20)$.

There were many clinical cases reported the misdiagnosis or coexistence between pelvic tuberculosis and ovarian cancer. Tuberculosis patients may present with peritoneal deposits, omental thickening, lymph node enlargement and raised CA-125 levels, hence mimicking ovarian cancer and incur unnecessarily extensive surgery, image guided percutaneous peritoneal biopsy and laparotomy are usually mandatory to distinguish these two entities. Our case is unique due to its a rare condition, that the lymphatic tuberculosis was misdiagnosed as lymphatic metastasis of ovarian cancer without tuberculous lesion were found in peritoneum. In our case, the patient had no history of TB or contact with TB patients and lacked typical symptoms and related signs of TB, with normal serum CA125, and imaging support for lymph node metastasis. Given the histological type, preoperative clinical stage, and imaging examination of ovarian cancer, intraoperative lymph node dissection was conducted.

Preoperative evaluation and intraoperative decisionmaking are very important to avoid excessive surgery and clarify the nature of enlarged lymph nodes. The most common progression of ovarian cancer involves intraperitoneal implantation and metastasis. Malignant tumor cells are implanted throughout the abdominal cavity 
(peritoneal organs) through intraperitoneal fluid circulation. If the focus is limited to one ovary, the probability of multiple pelvic and peritoneal lymph node metastases is low. Lymph node metastasis is rare, especially for the special pathological type of sex cord-stromal tumors. Although preoperative imaging examination may suggest multiple pelvic and abdominal lymph node metastasis, there are limitations of imaging examinations. Although the accuracy of PET/CT for the recognition of lymph node metastasis is better than that of CT and MRI, lymphatic TB must be considered for patients with lymph node metastasis diagnosed by PET-CT, especially in the epidemic areas of TB. If the nature of the enlarged lymph nodes cannot be determined before surgery, intraoperative sampling of the enlarged and/or suspicious lymph nodes can be performed and sent for intraoperative frozen pathological examination. In addition, we suggest that a multidisciplinary team composed of gynecological oncologists, physicians, radiologists, and radiation oncologists be established to conduct accurate preoperative evaluations and formulate effective treatment plans for these patients.

There has always been controversy regarding lymph node resection for ovarian cancer. There have been false positives using preoperative CT, MRI, and PET-CT evaluations of lymph node metastasis of ovarian cancer. Clinicians should therefore avoid relying exclusively on imaging results. Rather, they should make comprehensive decisions based on the patient's medical history, intraoperative conditions, and histopathological types. If necessary, clinicians can sample suspicious and enlarged lymph nodes, and intraoperative frozen pathological examinations can be conducted. By preventing a misdiagnosis, clinicians can therefore avoid excessive surgical treatments.

\section{Acknowledgments}

Funding: This work was supported by the Natural Science Foundation of China (No. 81960466).

\section{Footnote}

Reporting Checklist: The authors have completed the CARE reporting checklist. Available at https://gs.amegroups.com/ article/view/10.21037/gs-22-8/rc

Conflicts of Interest: All authors have completed the ICMJE uniform disclosure form (available at https://gs.amegroups. com/article/view/10.21037/gs-22-8/coif). The authors have no conflicts of interest to declare.

Ethical Statement: The authors are accountable for all aspects of the work in ensuring that questions related to the accuracy or integrity of any part of the work are appropriately investigated and resolved. All procedures performed in this study were in accordance with the ethical standards of the institutional and/or national research committee(s) and with the Helsinki Declaration (as revised in 2013). Written informed consent was obtained from the patient for publication of this case report and accompanying images. A copy of the written consent is available for review by the editorial office of this journal.

Open Access Statement: This is an Open Access article distributed in accordance with the Creative Commons Attribution-NonCommercial-NoDerivs 4.0 International License (CC BY-NC-ND 4.0), which permits the noncommercial replication and distribution of the article with the strict proviso that no changes or edits are made and the original work is properly cited (including links to both the formal publication through the relevant DOI and the license). See: https://creativecommons.org/licenses/by-nc-nd/4.0/.

\section{References}

1. Hernandez-Lopez LA, Elizalde-Mendez A. How far should we go in optimal cytoreductive surgery for ovarian cancer? Chin Clin Oncol 2020;9:70.

2. Gasimli K, Braicu EI, Nassir M, et al. Lymph Node Involvement Pattern and Survival Differences of FIGO IIIC and FIGO IIIA1 Ovarian Cancer Patients After Primary Complete Tumor Debulking Surgery: A 10-Year Retrospective Analysis of the Tumor Bank Ovarian Cancer Network. Ann Surg Oncol 2016;23:1279-86.

3. Bizzarri N, du Bois A, Fruscio R, et al. Is there any therapeutic role of pelvic and para-aortic lymphadenectomy in apparent early stage epithelial ovarian cancer? Gynecol Oncol 2021;160:56-63.

4. Ercelep O, Ozcelik M, Gumus M. Association of lymphadenectomy and survival in epithelial ovarian cancer. Curr Probl Cancer 2019;43:151-9.

5. Harter P, Sehouli J, Lorusso D, et al. A Randomized Trial of Lymphadenectomy in Patients with Advanced Ovarian Neoplasms. N Engl J Med 2019;380:822-32.

6. Ovarian Cancer Including Fallopian Tube Cancer and Primary Peritoneal Cancer. national comprehensive cancer network, Version 2.2020 - January 12, 2021. 
7. Chakaya J, Khan M, Ntoumi F, et al. Global Tuberculosis Report 2020 - Reflections on the Global TB burden, treatment and prevention efforts. Int J Infect Dis 2021;113 Suppl 1:S7-S12.

8. Norbis L, Alagna R, Tortoli E, et al. Challenges and perspectives in the diagnosis of extrapulmonary tuberculosis. Expert Rev Anti Infect Ther 2014;12:633-47.

9. Fontanilla JM, Barnes A, von Reyn CF. Current diagnosis and management of peripheral tuberculous lymphadenitis. Clin Infect Dis 2011;53:555-62.

10. Kitajima K, Murakami K, Yamasaki E, et al. Diagnostic accuracy of integrated FDG-PET/contrast-enhanced CT in staging ovarian cancer: comparison with enhanced CT. Eur J Nucl Med Mol Imaging 2008;35:1912-20.

11. Khiewvan B, Torigian DA, Emamzadehfard S, et al. An update on the role of PET/CT and PET/MRI in ovarian cancer. Eur J Nucl Med Mol Imaging 2017;44:1079-91.

12. Yuan Y, Gu ZX, Tao XF, et al. Computer tomography, magnetic resonance imaging, and positron emission tomography or positron emission tomography/computer tomography for detection of metastatic lymph nodes in patients with ovarian cancer: a meta-analysis. Eur J Radiol 2012;81:1002-6.

13. Tardieu A, Ouldamer L, Margueritte F, et al. Assessment of Lymph Node Involvement with PET-CT in Advanced Epithelial Ovarian Cancer. A FRANCOGYN Group Study. J Clin Med 2021;10:602.

Cite this article as: Sheng S, Chi B, Kuang Y. Lymphatic tuberculosis after lymphadenectomy for ovarian cancer: a case report. Gland Surg 2022;11(2):483-493. doi: 10.21037/gs-22-8
14. Kemppainen J, Hynninen J, Virtanen J, et al. PET/CT for Evaluation of Ovarian Cancer. Semin Nucl Med 2019;49:484-92.

15. Liu W, Li X, Yin J, et al. Diagnostic value of PET/ CT imaging in extrapulmonary tuberculosis. Journal of Southern Medical University, 2013;33:4.

16. Sathekge MM, Maes A, Pottel H, et al. Dual time-point FDG PET-CT for differentiating benign from malignant solitary pulmonary nodules in a TB endemic area. S Afr Med J 2010;100:598-601.

17. Levin G, Zigron R, Haj-Yahya R, et al. Granulosa cell tumor of ovary: A systematic review of recent evidence. Eur J Obstet Gynecol Reprod Biol 2018;225:57-61.

18. Erkılınç S, Taylan E, Karataşlı V, et al. Does lymphadenectomy effect postoperative surgical morbidity and survival in patients with adult granulosa cell tumor of ovary? J Obstet Gynaecol Res 2019;45:1019-25.

19. Brown J, Sood AK, Deavers MT, et al. Patterns of metastasis in sex cord-stromal tumors of the ovary: can routine staging lymphadenectomy be omitted? Gynecol Oncol 2009;113:86-90.

20. Saini A, Yadav G, Gothwal M, et al. Tuberculosis and ovarian malignancy: Sometimes mimics, sometimes coexists. J Obstet Gynaecol Res 2020;46:945-9.

(English Language Editor: L. Roberts) 